\title{
Collision induced spatial organization of microtubules
}

\author{
Vladimir A. Baulin, Carlos M. Marques, Fabrice Thalmann \\ Institut Charles Sadron CNRS UPR 22, 67083 Strasbourg Cedex, France
}

(Dated: October 31, 2018)

\begin{abstract}
The dynamic behavior of microtubules in solution can be strongly modified by interactions with walls or other structures. We examine here a microtubule growth model where the increase in size of the plus-end is perturbed by collisions with other microtubules. We show that such a simple mechanism of constrained growth can induce ordered structures and patterns from an initially isotropic and homogeneous suspension. First, microtubules self-organize locally in randomly oriented domains that grow and compete with each other. By imposing even a weak orientation bias, external forces like gravity or cellular boundaries may bias the domain distribution eventually leading to a macroscopic sample orientation.
\end{abstract}

\section{INTRODUCTION}

Biological processes like cell division, transport of certain organelles, morphogenesis and organization in the cell are mediated by rod like structures known as microtubules, which form various arrays, radial spindles, parallel and antiparallel bundles [1, 2, 3]. The microtubule self-assembly in living organisms is regulated by different factors: microtubule-associated proteins (MAPs) which stabilize, destabilize and crosslink microtubules 4, 5], diverse kinesin-like motor proteins, which organize and link microtubules, $\gamma$-tubulin ring complex which serves as a template for nucleation sites for microtubule polymerization in centrosomes 6,7 . These factors combine with the physical and chemical properties of the solution to determine, by mechanisms not yet well understood, the spatial structure and the orientation of the microtubules.

Each individual microtubule is a highly dynamic selfassembled rod, which is permanently growing or shrinking. This ability for being in an everlasting state of changing length as won microtubules the name of "searching devices" for specific targets in the cell [3, [8]. A key property allowing for this bistable state is the dynamic instability [4]. Due to conformational asymmetry of the constituting microtubule subunit, the heterodimer $\alpha, \beta$-tubulin, a microtubule has a polar structure and it grows at a plus-end and shrinks at its minus-end. The speed of growth at the plus-end is not constant, but rather intermittent. The elongation of the plus-end is stochastically alternated by the abrupt shrinking, in a process of unidimensional diffusion [9]. Such dynamic behavior is attributed to the complex, two-stage assembly of the plus-end, implying the internal hydrolysis of the GTP in a tubulin dimer. Within the cap model [4, 5, 10], the tubulins added to the growing plus-end are not hydrolyzed, thus having configurations favorable for the microtubule assembly. They form a cap protecting the plus-end from the disassembly and shrinking. Tubulins incorporated into microtubules are capable for hydrolysis. During conversion of the GTP of $\beta$-subunit to the GDP, the tubulin heterodimer undergoes the conformational change that destabilize the tubular structure of a microtubule and favors shrinking [1]. The rate of hydrolysis compete with the polymerization rate of the plus-end. Some factors like local fluctuations of tubulin concentration near the plus-end may perturb the balance thus leading to the loss of the protecting cap and provoking rapid shrinking. Since the density fluctuations have a stochastic nature, the growth to shrinking transitions (catastrophes) and the inverse resume of growth (rescue) are also statistically distributed.

The catastrophes can also be induced by factors other than concentration fluctuations. During the growing state, microtubules interact with each other as well as with different cellular structures. The mechanical force opposing the growth of the plus-end may induce catastrophes 12, 13. Experiments involving growing microtubules and different immobile obstacles and barriers have shown that the opposing force reduces the growth velocity [14, 15]. Thus, the dynamic instability coupled with the mechanical force may influence the microtubule length and thus induce a spatial organization. Indeed, the catastrophe rate is expected to be higher near cellular boundaries and lower in the cytoplasm [12]. The boundaries may also induce the orientation preference, since the catastrophe rate in the direction perpendicular to the boundary would be higher then that along the boundary. Recent in-vivo work suggests that the microtubule dynamic instability is altered during preprophase band formation [16]. Microtubule reorientation is accompanied by the increase of the catastrophe frequency and growth rate, while the rescue frequency and shrinkage rate remain unchanged. The gradients always present in living cells can also play a role of the "effective" boundaries and provoke the microtubules ordering [17]. In particular, the gradients of the energy dissipation, concentration of tubulin and associated proteins may result in spatial anisotropy of the growing and shrinking speeds which can lead to a self-organization.

Another important observation concerns the intermicrotubules interactions 18]. Encounters between cortical microtubules affect their dynamic behavior. Steep contact angles of microtubules collisions provoke catastrophes more often than the shallow contact angles, while the microtubules with close angles have shown a tendency to zippering.

These observations suggest that the spatial self- 
organization of microtubules might be induced by the coupling of the catastrophe events and the configuration of neighboring microtubules. Different examples of spontaneous self-organization of dynamic microtubules have been reported in the literature [19, 20, 21, 22, 23]. One of them [20], describes the formation of spatial structures in the in-vitro solution of microtubules that start growing from seeds distributed homogeneously. The resulting pattern strongly depends on the direction of the gravitational field, where microtubules are organized as highly aligned strips. Interestingly, the observed structures do not appear in weightlessness conditions. The authors conclude from their observations that the Earth gravity only triggers the symmetry breaking and does not affect individual microtubules. The concentration of microtubules even coupled with gravity is not sufficient to provoke the system orientation due to excluded volume effects as in usual liquid crystals [24, 25]: the shaking or mixing of the sample irrevocably destroys the pattern. Since the in-vitro preparation does not contain any molecular motors or MAPs, the self-organization in stripes is attributed to the dynamic nature of the microtubules: the pattern formation disappears when the dynamic instability is inhibited by the addition of taxol. Similar patterns are formed under magnetic fields [21]. The in-vivo observations of the reorientation of cortical microtubules in parallel arrays [22, 23, 26, 27] are also ascribed to dynamic instability: the depolymerization of disordered microtubules is followed by the repolymerization into an ordered array. However, the in-vivo selforganization can be regulated by MAPs or motor proteins [7, 28] or it may be a result of the simultaneous

A microtubule is modeled as a rigid, oriented rod which shrinks at its minus-end and grows at its plusend. Instead of dealing with a fluctuating rate of growth and shrinking, we rather consider smooth, coarse-grained properties, namely the average speeds of growth and shrinking. Without obstruction, in a free environment, the plus-end of a microtubule grows at constant speed $v^{+}$, while its minus-end shrinks at constant speed $v^{-}$. When the plus-end encounters another rod, it stops, but the rod continues to shrink at its minus-end, with speed $v^{-}$, and as a result, the overall length of the rod decreases. The rod resumes its growth as soon as its plus-end is no longer blocked by its neighbor. Altogether, the plus-end experiences an environment dependent intermittent growth, and the minus-end a constant motion at speed $v^{-}$. The rod disappears if its length decreases to zero during the shrinkage phase. The total number of rods is not fixed, but is maintained by a permanent injection rate of new rods at random positions, with random orientation and zero length.

We found that this mechanism of the constrained growth can lead itself to the spontaneous alignment of microtubules in an initially isotropic and homogeneous array. The emergence of a local orientational order is reminiscent from a natural selection process. Whenever some local anisotropy builds up, the survival rate of the action of many factors.

In this paper we explore the consequences of the simplest possible physical hypothesis for explaining microtubule orientation from a coupling mechanism between growth and inter-tubule interactions. In the next section we set the foundations for the physical model based on the observation that the inter-microtubules collisions increase the rate of catastrophes [18]. In section III we show numerically that this mechanism alone leads to the orientation of microtubules in aligned stripes. A theoretical discussion of our main results is presented in section IV and our findings are summarized in the conclusion.

\section{THE MODEL}

\section{A. A kinetically constrained growth model}

We propose a model based on the assumption that the assembly dynamics of a particular microtubule is influenced by the configuration of the surrounding microtubules. Our purpose is to exhibit the simplest possible model of constrained growth inducing some selforganization of the microtubules. Thus, in our model, we neglect all the other possible physical mechanisms that could play a role in the co-alignment, such as collision induced turnover and dislocation 22], as well as the excluded volume interactions leading to ordering in usual liquid crystals. The incorporation of these factors would facilitate and enhance the alignment.

neighboring rods changes and becomes orientation dependent. The rods which from the start have picked up the dominant orientation are likely to outlive rods with a different orientation, and this creates conditions favoring the population of rods with the "correct" orientation at the expense of rods with "incorrect" orientation. In our model, the rods cannot change their orientation but the permanent injection of young, randomly oriented rods, leaves to the system the possibility to reorganize and to tune up to a change of external conditions.

We implemented numerically this constrained growth model. The simulations clearly show a trend towards some local organization and ordering, with the formation of well defined anisotropic domains.

\section{B. Numerical implementation of the constrained growth model}

In our numerical implementation, in two dimensions, each rod is characterized by its length, its position, and its orientation. The orientation (one angle) and the position (two coordinates) are set when the rod is injected, and do not change until the rod eventually disappears. The length of each rod evolves with time, starting from zero shortly after the injection. All rods are packed in 

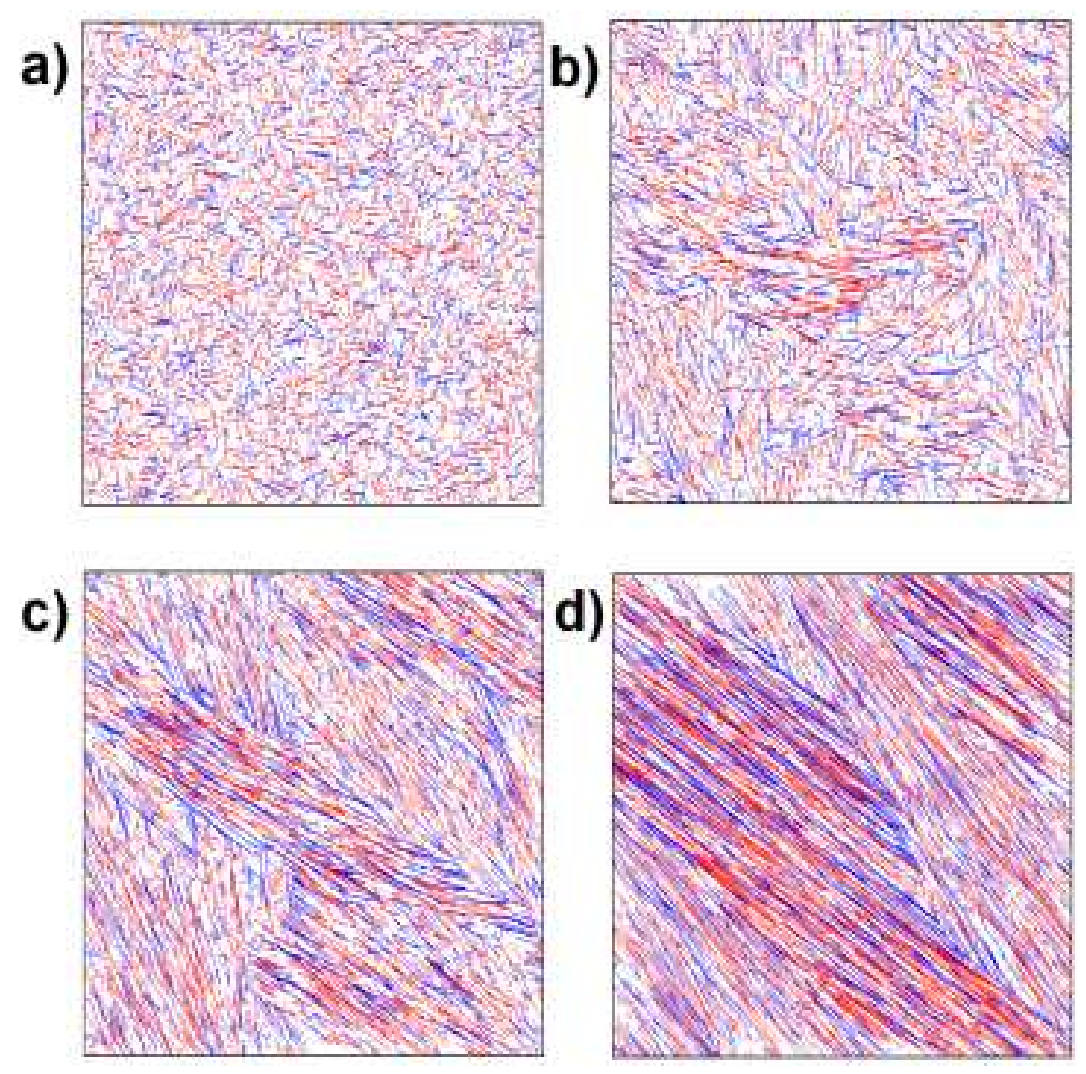

FIG. 1: Snapshots of the system of rods at various successive times (time increases from a to d). Growing rods are drawn in blue, and shrinking rods in red.

a square box of side $L_{s}$, subject to periodic boundary conditions.

Depending on their dynamic state, the rods belong to two categories: shrinking rods ( $s$-rods) are the rods that the local environment prevents from growing further (kinetic constraint), while growing rods ( $g$-rods) are free to grow.

The rule for the time evolution of the rods is the noncrossing displacement. Updates are done every time interval $\Delta t$. The minus tip of each rod is shortened by the amount $v^{-} \Delta t$, while the plus tip attempts a move forward by $v^{+} \Delta t$. If this move can be done without crossing any other rod, the move is accepted and the rod grows. If the rod was in a $s$-rod state, it converts to a $g$-rod state. Otherwise, the move is rejected, and the rod switches to, or stays in a blocked $s$-rod state. As a result, the length of a rod after each step, either increases by $\left(v^{+}-v^{-}\right) \Delta t$, or decreases by $v^{-} \Delta t$.

In the present case, we chose the values $L_{s}=100$ and $\Delta t=1$. The speed $v^{+}$ranges from 0.5 to 2.1 and $v^{-}=$ 0.3. In the discussion, we make a frequent use of the speed ratio $\alpha$ :

$$
\alpha=\frac{v^{-}}{v^{+}-v^{-}}
$$

defined as the ratio between the speed of shrinkage in the $s$-state $v^{-}$and the speed of growth in the $g$-state $v^{+}-v^{-}$. The corresponding values of $\alpha$ used in the simulation lie in the interval 0.17 to 1.5 . The value for the injection rate $Q_{i}$ is about 1000 new rods per unit of time. There are typically a few thousand rods (5000 to 6000) at a time in the simulation box.

\section{MAIN RESULTS}

\section{A. Spatial organization: domain structure}

The simulation starts with a set of rods of zero length. The kinetic constraint concerns a vanishingly small number of rods at the early stage of the system evolution. As the number and the length of the rods increase, the amount of packing gets larger, and the kinetic constraint forces a significant fraction of rods into a blocked, shrinking state. This transient regime recedes to a quasistationary regime in which the ratio of $s$-rods and $g$-rods seems to remain approximately constant.

Then, the numerical simulations shows clearly the slow emergence of oriented domains, or bundles, of nearly aligned rods. These domains show very rough, ragged and sharp boundaries, much as crystallites. Thus, they look quite different from the domains arising in the usual 


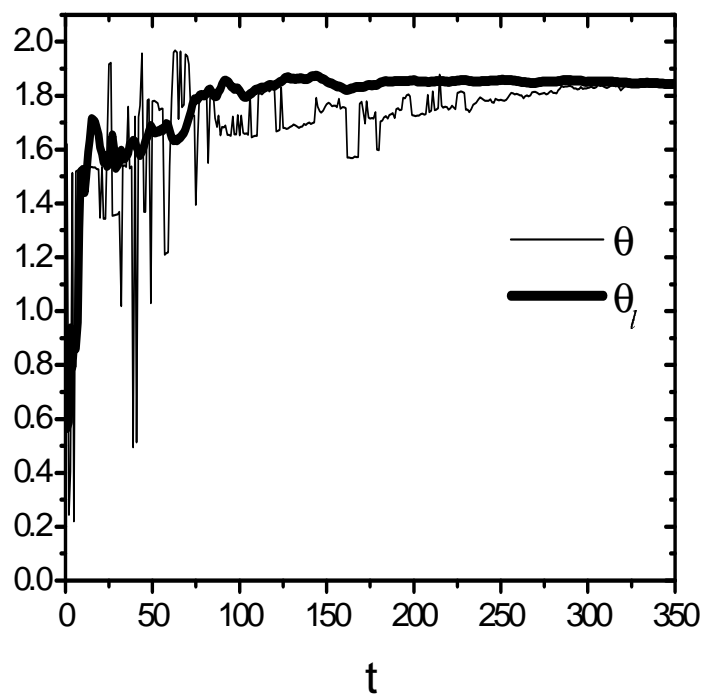

FIG. 2: The dominant angles $\Theta, \Theta_{l}$ as a function of the time, as found by minimizing equations 3 and 6

phase transitions and coarsening situations, where a finite bending elasticity creates a smooth variation of the order parameter, at the vicinity of a domain wall. The domains seems to be randomly oriented, and the isotropy of the system is recovered only on length scales larger than the size of the domains.

In the final stages of the simulation, the average domain size $L(t)$ is still a slowly growing function of the time, and eventually becomes of the same order of magnitude as the size of the system $L_{s}$. This prevents reaching an asymptotic finite value of $L(t)$, associated to a truly stationary distribution of the rod lengths and orientations. Well known examples of such coarsening dynamics are characterized, for instance, by a power-law or a logarithmic behavior of $L$ with $t$ [29]. In the latter case, the possibility to discuss the system properties in term of quasi-stationary solutions remains.

Figure 1 illustrates how the rods self-organize with time. At first, the population of rods is isotropic, except for small fluctuations inherent to the random initial position and orientation distribution (Figure 1a). These pre-existing heterogeneities grow into small bundles, whose distribution still remain seemingly isotropic on large scales (Figure 1b). Then, larger bundles emerge at the expense of many other smaller bundles, bound to disappear (Figure 1). Finally, the typical size of the larger bundles becomes comparable to the size of the simulation box (Figure 11). The absence of bending modulus, and the presence of ragged boundaries, forbids a mechanism based upon domain walls motion. Such a competitive growth of the domains is slow.

To quantify the degree of local ordering, or "polarization", of the system, we introduce a dominant angle $\Theta$,

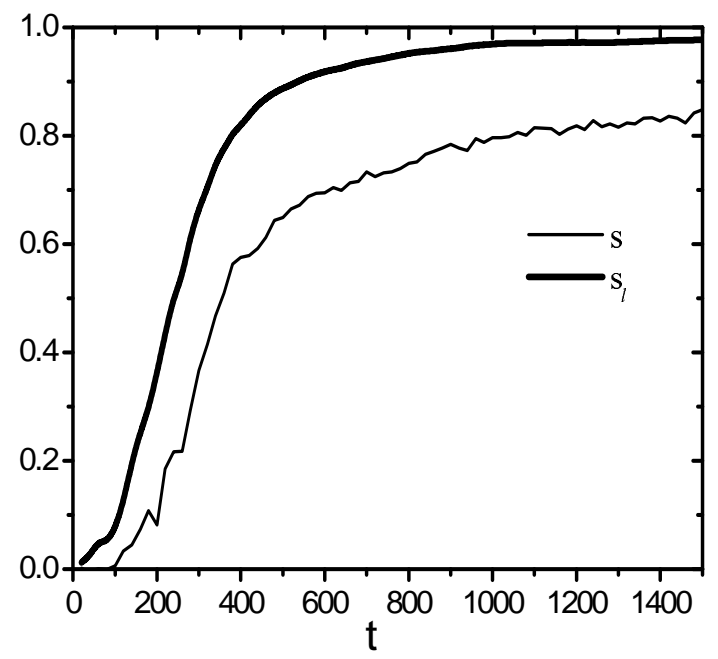

FIG. 3: Anisotropy ratios $S_{l}$ and $S$ vs time $t$.

which maximizes a cost function $\sigma$ :

$$
\begin{aligned}
\sigma(\theta) & =\frac{1}{n_{t}} \sum_{i=1}^{n_{t}} \cos ^{2}\left(\Omega_{i}-\theta\right), \\
& =\overline{\cos ^{2}(\Omega-\theta)}
\end{aligned}
$$

where $\Omega_{i}$ is the orientation (angle) of the $i^{t h} \operatorname{rod}$, and the sum runs over $n_{t}$, the total number of rods present in the system. Thus, $\sigma$ is defined as the "ensemble average" over the population of rods at time $t$, denoted with an overline $\ldots$ We call order parameter the value of the maximum $s=\sigma(\Theta)$. The cost function can be expanded as $\sigma(\theta)=\overline{\cos ^{2} \Omega} \cos ^{2} \theta+\overline{\sin ^{2} \Omega} \sin ^{2} \theta+\overline{2 \sin \Omega \cos \Omega} \sin ^{2} \theta$. Then, the stationarity condition $\partial \sigma(\theta) /\left.\partial \theta\right|_{\Theta}=0$ leads to:

$$
\tan 2 \Theta=\frac{\overline{\sin 2 \Omega}}{\overline{\cos 2 \Omega}} .
$$

This equation has always four solutions, two corresponding to the maxima $\Theta_{\max }$ and $\Theta_{\max }+\pi$, and the other two, to the minima $\Theta_{\min }$ or $\Theta_{\min }+\pi$, and $\Theta_{\max }$ and $\Theta_{\min }$ are mutually orthogonal.

A scaled anisotropy parameter $S$ may be defined as:

$$
S=\frac{\sigma\left(\Theta_{\max }\right)-\sigma\left(\Theta_{\min }\right)}{\sigma\left(\Theta_{\max }\right)+\sigma\left(\Theta_{\min }\right)} .
$$

The quantity $S$ is 0 for a population of isotropically oriented rods, while it is 1 for a population of perfectly aligned rods. The parameter $S$ makes it possible to quantitatively assess the amount of ordering in the system. Because both parameters $s$ and $S$ turn out to fluctuate strongly with time, we introduce a more stable parameter, where each rod $i$ contributes according to its length $l_{i}$ :

$$
\sigma_{l}(\theta)=\frac{1}{n_{t}} \sum_{i=1}^{n_{t}} l_{i}^{2} \cos ^{2}\left(\Omega_{i}-\theta\right),
$$




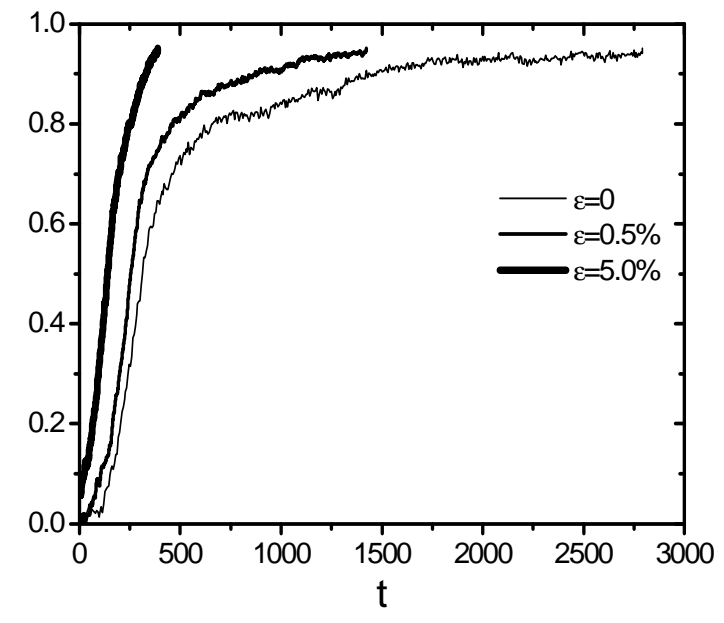

FIG. 4: The anisotropy ratio $S_{l}$ vs time, for three increasing biases $\epsilon$ in the angular distribution of the rods orientation.

where the long rods participate more than the short ones. The dominant angle associated with this parameter obeys:

$$
\tan 2 \Theta_{l}=\frac{\overline{l^{2} \sin 2 \Omega}}{\overline{l^{2} \cos 2 \Omega}}
$$

and the anisotropy ratio $S_{l}$ defined as in Eq. 4 with $\sigma$ replaced by $\sigma_{l}$.

Figure 2 shows the variation of the dominant angles $\Theta$ and $\Theta_{l}$ with time, for one set of parameters. At the beginning, the system is homogeneous and the distribution of angles is isotropic, resulting in a singular and noisy function of time. As the system evolves, the ordered structures appear and the angles stabilize around their preferred value. It is noteworthy that the $\Theta_{l}$ curve is smoother than the $\Theta$ curve, due the stabilizing contribution of the longest and most stable rods. The plateau value is related to the orientation of the dominant bundle, and fluctuates from sample to sample.

The same conclusion can be drawn from the plot of the anisotropy ratios $S$ and $S_{l}$, function of time in Figure 3. Although evolving on the same time scale as $S$, the quantity $S_{l}$ reaches a value closer to 1 . The differences between the two curves is most certainly due to the contribution of the many young, short rods, upon which the kinetic constraint has not been acting long enough to force them into the dominant orientation.

The anisotropy ratio and the dominant angles aim at quantifying the degree of local ordering in a suspension of rod like objects, irrespective of the underlying alignment mechanism. In that respect, they make possible a direct comparison with other alternative models of microtubule orientation. From these curves, one can infer a characteristic time $t^{*}$ for the emergence of a global orientation in the sample, such as, for instance, $S_{l}\left(t^{*}\right)=1 / 2$. In Figure 3. this ordering time is about a few hundred steps $\left(t^{*} \sim 300\right)$.

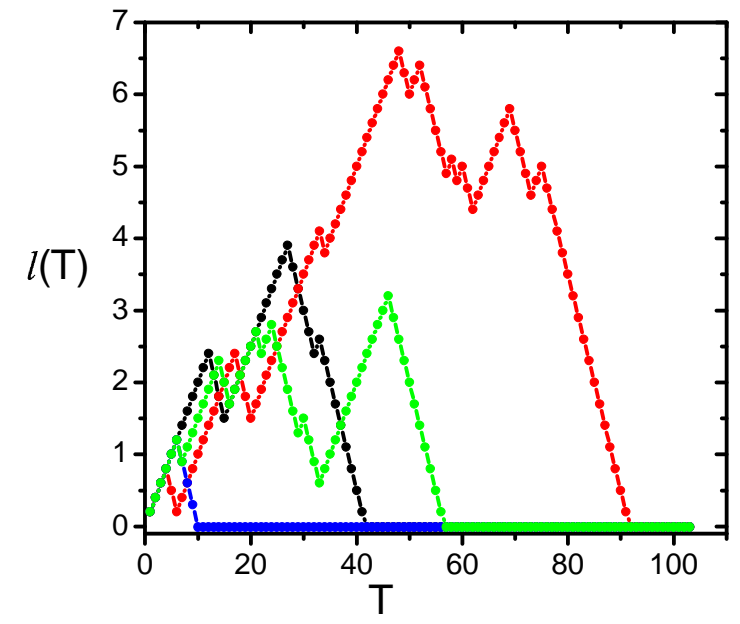

FIG. 5: Different rod histories, showing the length $l(t)$ function of the time $t$. The curves are similar to random walks with an absorbing boundary condition at $l=0$.

\section{B. Sensitivity to external stresses}

The constant renewal of the rods, along with the growth of the competing domains, confers to the system the ability to respond to external perturbations. One of our main motivation is to evaluate the sensitivity of the ordering to the presence of an external gravitational, or magnetic field. Quite similarly, the presence of a hard wall is expected to align the nearby domains along its direction.

In order to probe the ability of the rods suspension to cope with external constraints, and to monitor its susceptibility to a small symmetry breaking, we performed several simulations with a slightly biased distribution in the orientation of the newly injected rods. Instead of being isotropic, we added a fraction $\epsilon$ of rods in excess, with an angle $\Omega$ belonging to a small interval $\Omega_{0} \pm 1^{\circ}$. As a result, a value $\epsilon=0.5 \%$ brings about an acceleration of the ordering time $t^{*}$ by a factor 1.5 , and a value $\epsilon=5 \%$ triggers a three times faster growth of the domains (Figure (4). In both cases, the preferred orientation is clearly related to the orientation of the bias $\Omega_{0}$.

Boundaries and impurities can also induce the alignment. When one of the periodic boundary conditions is replaced by a hard wall, the alignment of the rods is much faster, and the wall orientation propagates into the bulk of the suspension. An identical behavior is observed when a rod with fixed length, position and orientation, is forced into the simulation box. The rods orient themselves parallel to the guiding rod, and longer guiding rods provoke faster ordering. 


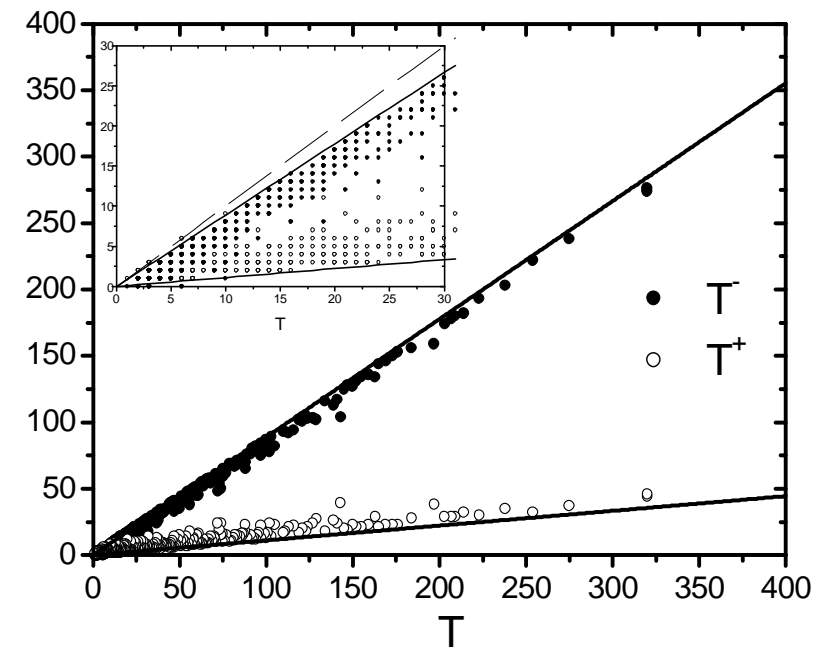

FIG. 6: Scatter plot of the times of growth $T^{+}$and $T^{-}$, as a function of the total age $T=T^{+}+T^{-}$, for a given population at time $t$. Inset: a close-up look at the region of young rods.

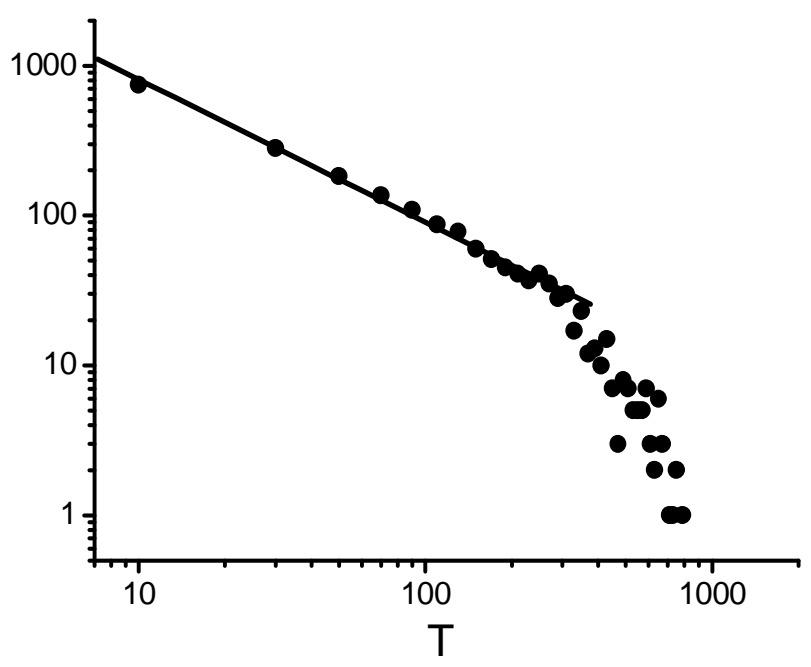

FIG. 7: Histogram of the distribution of the ages $T$ of a population of rods, in logarithmic coordinates. The initial decay rate is close to $1 / T$, followed by an exponential decay.

\section{Kinetics of individual rods}

The numerical simulation makes it possible to track a single rod as it evolves with time. We observe that during its life cycle, a rod can experience many alternating periods of growth and shrinkage. A typical microtubule life history plot is shown in Figure [5] following a saw-teeth curve.

We call "age" $T$, the time interval elapsed since the rod was injected with zero length in the system. The age is the sum of the growing time $T^{+}$and the shrinking time $T^{-}$, and the length of the rods can be expressed with the

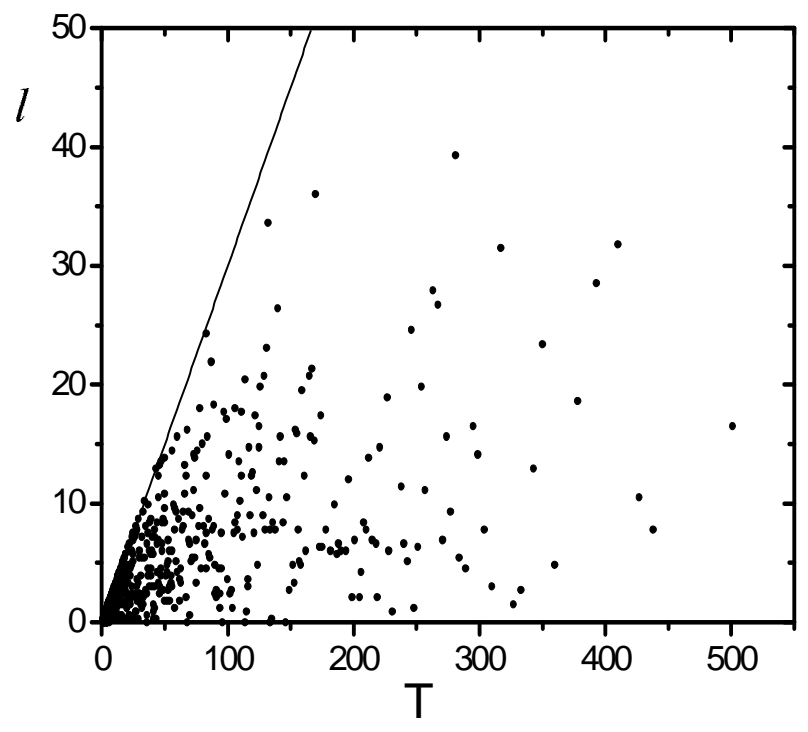

FIG. 8: Scatter plot of the length (vertical axis) and the age (horizontal axis).

help of $T^{+}, T^{-}$as

$$
\begin{aligned}
l & =\left(v^{+}-v^{-}\right) T^{+}-v^{-} T^{-} \\
T & =T^{+}+T^{-}
\end{aligned}
$$

or equivalently,

$$
T^{-}=\frac{\left(v^{+}-v^{-}\right) T-l}{v^{+}} ; T^{+}=\frac{v^{-} T+l}{v^{+}} .
$$

A typical distribution of both $T^{+}$and $T^{-}$, as a function of the age $T$, is shown in Figure 6 for a population of rods at a given time $t$ (scatter plot), while the inset of Figure 6] is an enlargement of this plot in the small $T$ region. The values $T^{+}$and $T^{-}$of old rods (large $T$ ), concentrate near two boundaries, which correspond to a length $l=0$ in Eq. 8 . For these old rods, which have survived many collisions, the growth and shrinkage periods compensate almost exactly, and in Eq. 7 the length $l$ results from the difference between two large quantities. By contrast, the young rods (small $T$ ) show all possible combinations of $T^{+}$and $T^{-}$(inset in Figure 6). This indicates that the young rods have not yet been influenced by their surrounding. The maximal possible length of a rod occurs in the extreme case $T^{-}=0$ and $T^{+}=T$, thus corresponding to a length $l_{\max }=\left(v^{+}-v^{-}\right) T$ (the dashed line in the inset of Figure 6).

Young rods enjoy a fast growth rate, but many are also eliminated quickly. Older rods show a smaller average growth rate, but their survival rate increases with their age. This is clear from the histogram of the ages, which is clearly not exponential, but rather well approximated by a power law $p_{s}(T) \sim T^{-1}$ (Figure 17). The relative disappearance rate of rods aged $T$, is $p_{s}^{-1} \mathrm{~d} p_{s} / \mathrm{d} t \sim T^{-1}$, instead of remaining constant, as in the exponential case (e.g like for instance the decay of radioactive elements). 

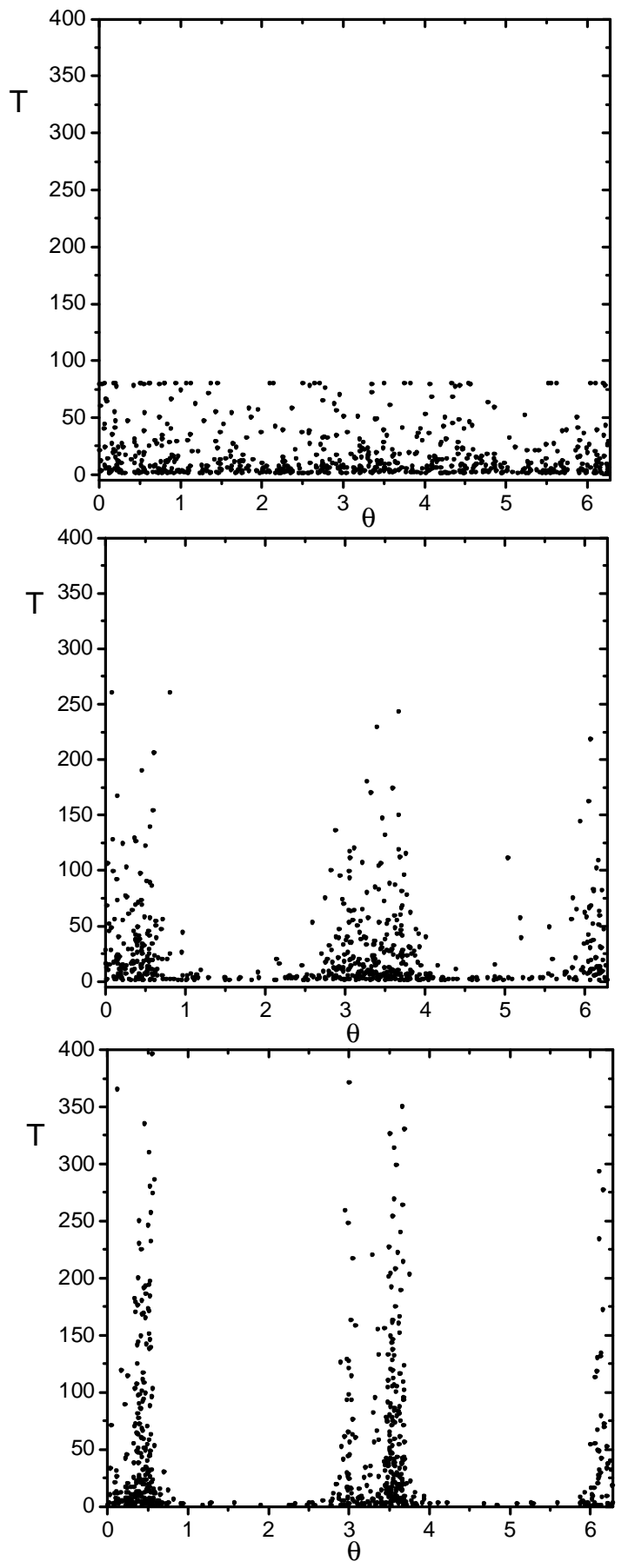

FIG. 9: Scatter plot of the orientation (horizontal axis) and the age (vertical axis) of the rods at three different stages of the evolution of the system. The time $t$ increases from top to bottom.

In a sense, the young rods shows "plastic" properties, and account for the adaptability properties of the rods suspension. By contrast, older rods are expected to show more rigidity and persistence from the past history of the suspension. We did not find any simple justification for the exponent -1 , which is a numerical finding. This con-

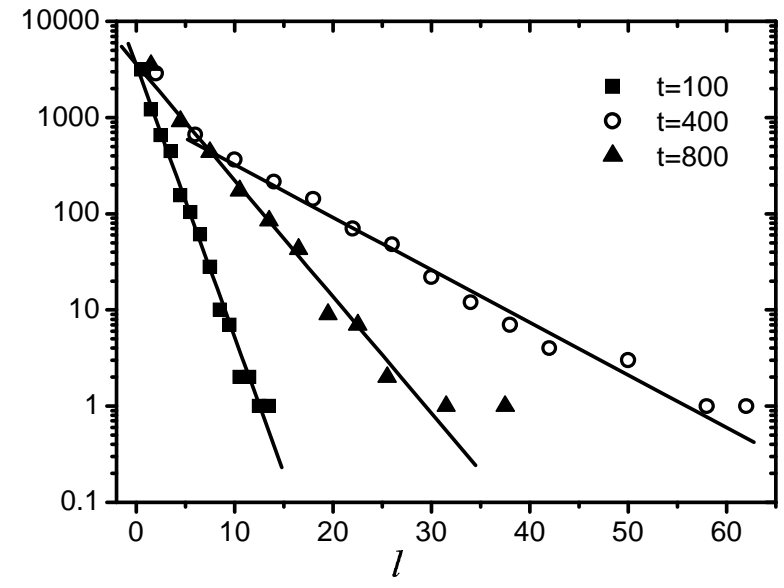

FIG. 10: Semilogarithmic plot of the histogram of the number of rods, for three different stages of the evolution of the system. Straight lines correspond to an exponential decay.

tradicts a naive argument based on the usual random walks, which would predict a $T^{-3 / 2}$ behavior associated to the first return to the origin time distribution. We still believe in the analogy, but we attribute this discrepancy to the existence of strong correlations between orientation, age and life time of the rods. Finally, the age of the very old rods is distributed exponentially (Figure 7 ).

A scatter plot of the lengths $l$ versus the ages $T$ of a population of rods does not support the presence of strong correlations between these two parameters (Figure 8).

\section{Distribution of lengths and orientations}

The ordered structures (bundles or domains) effectively select the rods, keeping only those with an orientation compatible with the dominant orientation of the bundles. The correctly oriented rods collide less often with their neighbors than the rods with transverse orientations, and their "fitness", or survival ability is greater.

Figure 9 shows three typical scatter plots of the ages as a function of the angles. The age of the system increases from the top to the bottom plot. The presence of anisotropic domains manifests itself as sharp peaks around a few well defined angles. On the example shown, one can see two different domains, respectively around $25^{\circ}$ and $160^{\circ}$. The peaks are duplicated (mirrored) because the bundles contain a mixture of two antiparallel populations, separated by $180^{\circ}$.

A typical distribution of lengths regardless to the rods orientation is shown in Figure 10. The distribution is exponential except for a very small region of tiny lengths. Most of the rods with tiny lengths are very young rods injected in the system a few steps ago. They did not have time to experience collisions and their distribution did not acquire the same characteristics as the old rods. 
The system not only adjusts the orientation and the length of the rods, but it also regulates the total number of rods $n_{t}$. An increase in the rods density leads to a higher collision rate among rods, and a higher elimination rate. In the early stage of the simulation, in a sparse system, the newly injected rods do not meet any obstacles and the total number of rods increases sharply. After a transient regime, $n_{t}$ evolves very slowly, although it is not strictly constant. As a matter of fact, $n_{t}$ slightly decreases with the spreading of the dominant orientation and the emergence of domains (Figure 11).

\section{E. The three dimensional case}

Finally, we performed a limited number of simulations in three dimensions, in order to check whether the kinetic ordering was a special feature of the two dimensional systems, or whether it was a generic feature also in three dimensions. In this case, our simulation box is a cube of size $100 \times 100 \times 100$. In addition to the injection rate $Q_{i}$, to the speeds $v^{+}$and $v^{-}$, there is another relevant parameter: the diameter, or thickness, of the rods $d$.

It turns out that the behavior of the system in three dimensions is quite similar to the one observed in two dimensions. The initially homogenous solution becomes gradually structured into bundles and domains. However, the three dimensional system differs by the absence of sharp boundaries between domains. The competition between the different orientations is not so drastic, since bundles with different orientation can interpenetrate if the rod thickness is small. Domain walls are more difficult to identify, but the main result, i.e. local ordering, holds also in three dimensions.

\section{THEORETICAL DISCUSSION}

We discuss in this part some observed features of our numerical simulations: exponential tails in the length distribution, collision rates, anisotropy. For this purpose, we propose an elementary kinetic theory, and its predictions are compared with the numerical simulations.

\section{A. Ensemble and time averages}

Much like in the usual statistical mechanics, one introduces two kinds of averages. The time average, denoted by brackets $\langle\ldots\rangle$ corresponds to the mean value obtained by the repeated observation of single rods evolving with time. For instance, $\left\langle T^{+}\right\rangle$is the average growth time of a rod. Time averages are accessible through numerical simulations.

The ensemble average amounts to considering the whole population of rods at a given time. This corresponds to an instantaneous "snapshot" of the population of rods, and the corresponding average is denoted with

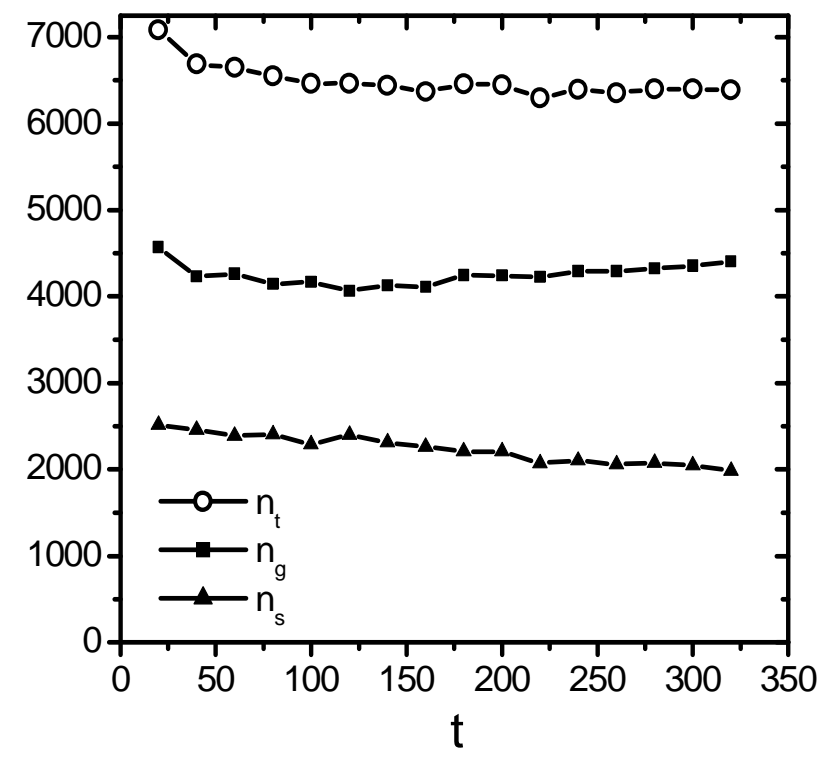

FIG. 11: Evolution of the number of rods $n_{t}$ with time, and repartition between shrinking rods $\left(n_{s}\right)$ and growing rods $\left(n_{g}\right)$.

an overline $\bar{\ldots}$ : for instance, $\bar{l}$ is the average length of the rods. In practice, the ensemble average consists in summing over all the rods, and then dividing by their total number $n_{t}$. The ensemble averages can be computed from the simulations, but are also the natural outputs of the kinetic theory sketched below.

The connection between time and ensemble average is by no means obvious. In the case of a true stationary situation, both averages are expected to coincide. Our situation, however is not a full stationary situation, as we witness the emergence of unbounded large bundles. A slow coarsening dynamics, however, may still exhibit a satisfactory agreement between the two kinds of averages.

Ensemble averages are conveniently handled by means of distribution functions. Denoting the length, orientation and position of the rods respectively by $l, \Omega$ and $\vec{r}$, we define $c_{t}(l, \Omega, \vec{r}, t)=c_{g}(l, \Omega, \vec{r}, t)+c_{s}(l, \Omega, \vec{r}, t)$, where $c_{g}, c_{s}$ and $c_{t}$ stand respectively for the distribution of the population of growing rods, shrinking rods and total number of rods, per unit of surface, at time $t$, with $0 \leq l<\infty, 0 \leq \Omega<2 \pi$ and position $\vec{r}$.

Successive integrations over the variables $\vec{r}, l$ or $\Omega$, give rise to a hierarchy of distribution functions. In particular, the numbers of rods $n_{s}$ and $n_{g}$ are given by:

$$
\begin{aligned}
& n_{g}(t)=\int \mathrm{d} \vec{r} \mathrm{~d} l \mathrm{~d} \Omega c_{g}(l, \Omega, \vec{r}, t) \\
& n_{s}(t)=\int \mathrm{d} \vec{r} \mathrm{~d} l \mathrm{~d} \Omega c_{s}(l, \Omega, \vec{r}, t) .
\end{aligned}
$$

In what follows, we use a loose notation for the partial distribution functions, where the variables which do not explicitly appear in $c_{g}$ have been implicitly integrated over, e.g. $c_{g}(l, \Omega) \mathrm{d} l \mathrm{~d} \Omega$ stands for the fraction of $g$-rods, 


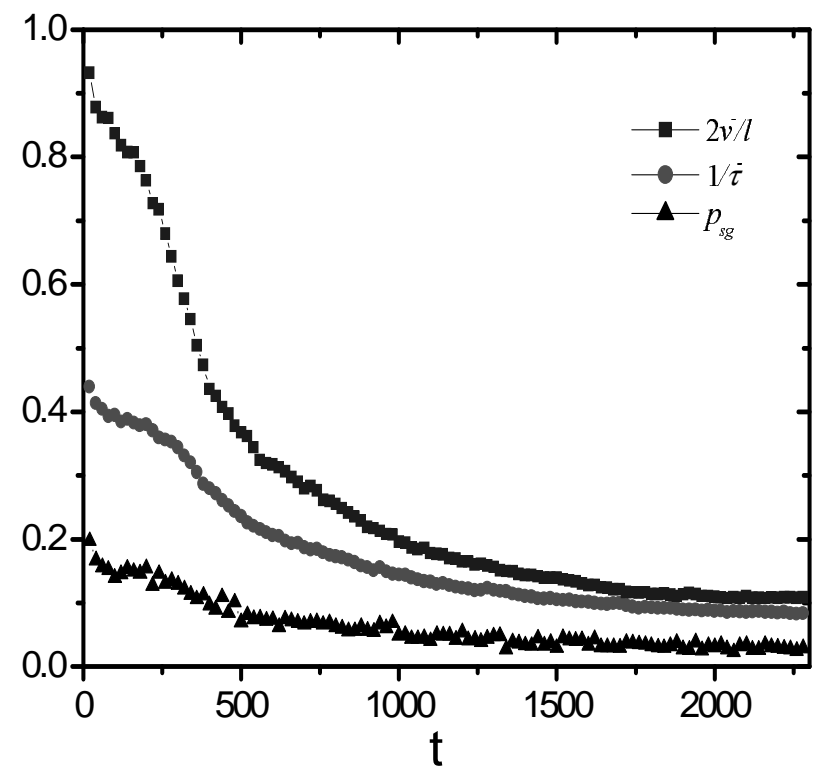

FIG. 12: Test of the relations Eq. 14 and Eq. B6] Circles: inverse of the average shrinkage interval $\left\langle\tau^{-}\right\rangle$, last term of Eq. 18 squares: r.h.s of Eq. 14 triangles: computed value of $p_{s g}$.

with length between $l$ and $l+\mathrm{d} l$, angle between $\Omega$ and $\Omega+\mathrm{d} \Omega$, but located at any position $\vec{r}$ of the system.

\section{B. The distribution of lengths}

Following the lines of Appendix A we restrict ourselves to the homogeneous, $\vec{r}$-independent case, and assume that the distributions $c_{g}$ and $c_{s}$ comply with the following master equation:

$$
\left\{\begin{array}{c}
\frac{\partial}{\partial t} c_{g}+v \frac{\partial}{\partial l} c_{g}=p_{s g} c_{s}-p_{g s} c_{g} \\
\frac{\partial}{\partial t} c_{s}-\alpha v \frac{\partial}{\partial l} c_{s}=p_{g s} c_{g}-p_{s g} c_{s}
\end{array}\right.
$$

The properties of the distributions mostly depend on the injection rate $Q_{i}$, the speed $v=v^{+}-v^{-}$, and the speed ratio $\alpha=v^{-} /\left(v^{+}-v^{-}\right)$. We introduce the interconversion rates $p_{s g}(\Omega)$ and $p_{g s}(\Omega)$ between $s$ and $g$ states, and we take care of a possible dependence on the direction $\Omega$. We find that, for an homogeneous and stationary system, the lengths are exponentially distributed and verify:

$$
\begin{aligned}
c_{g} & =\frac{Q_{i}}{v} \exp [-l / \bar{l}(\Omega)] ; \\
c_{s} & =\frac{Q_{i}}{\alpha v} \exp [-l / \bar{l}(\Omega)] .
\end{aligned}
$$

Such an exponential distribution can be seen in Figure 10 This model accounts for the possibility of anisotropic length distributions, via the angle dependent function $\bar{l}(\Omega)$. It is possible to find an isotropic, selfconsistent solution for $\bar{l}(\Omega)=\bar{l}$, but we found also evi-

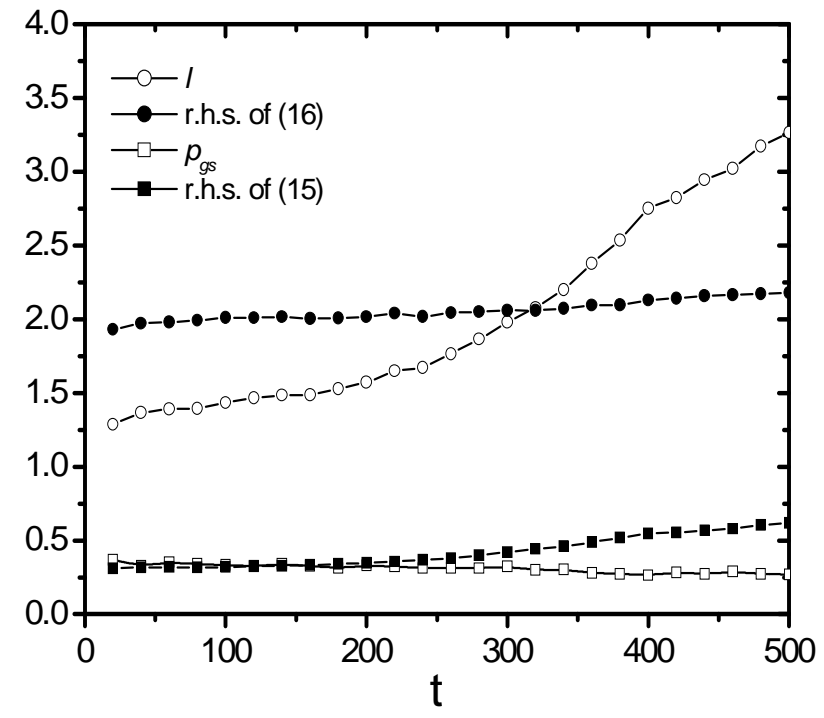

FIG. 13: Test of the relations Eq. 15 and Eq. 16 Open circles: $\bar{l}$, filled circles: r.h.s of Eq. [16] open squares: $p_{g s}$, filled squares: r.h.s of Eq. 15

dence for an anisotropic self-consistent solution, with a non trivial function $\bar{l}(\Omega)$.

Our predictions for the isotropic solution include a determination of the rate $p_{s g}$ :

$$
p_{s g}=\frac{2 v^{-}}{\bar{l}},
$$

a determination of the rate $p_{g s}$ :

$$
p_{g s}(\Omega)=v^{+} \frac{2 \bar{l}}{\pi}\left(\frac{n_{t}}{S}\right)
$$

and a self-consistent determination of the average length $\bar{l}$, as a function of $Q_{i}, \alpha$, the total number of rods $n_{t}$ and the surface $S$ :

$$
\bar{l}=\sqrt{\frac{3 \pi}{2(\alpha+1)} \frac{S}{n_{t}}} \simeq\left(\frac{S}{n_{t}}\right)^{\frac{1}{2}} .
$$

The predictions of Eq. 14 are shown in Figure 12 and discussed also in Appendix B. Agreement is poor for short times and it improves for long times.

The predictions of Eq. 15] and Eq. 16] are summarized in Figure 13. The agreement is good for $p_{g s}$ and qualitative for $\bar{l}$ at short times. The prediction becomes poor for times larger than $t^{*}$, associated to the emergence of the domains. We believe that the disagreement is mainly due to the impossibility for a two-dimensional system to be at the same time considered as anisotropic and homogeneous. By contrast, this would be a more reasonable assumption in a three dimensional space. Our kinetic theory shows too strong mean-field features to be able to describe accurately this two-dimensional situation. We conclude that the predictions of this isotropic model are no longer valid when the domains start growing. 


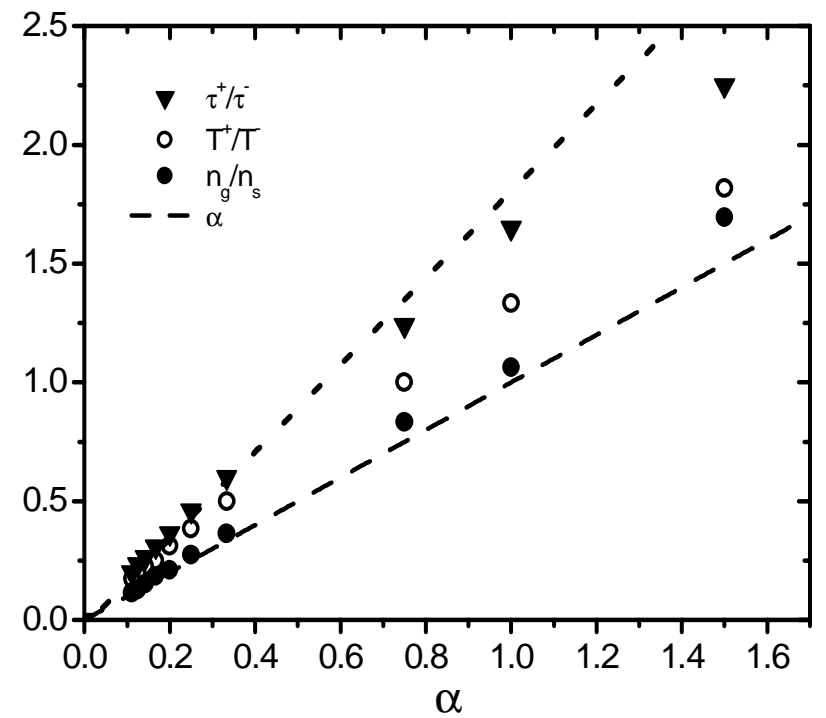

FIG. 14: Horizontal axis: speed ratio $\alpha=v^{-} /\left(v^{+}-v^{-}\right)$. Various curves are represented: the ratio of the times of growth to the times of shrinking $\left\langle T^{+}\right\rangle /\left\langle T^{-}\right\rangle$, the ratio of intervals of growth and the intervals of shrinking $\left\langle\tau^{+}\right\rangle /\left\langle\tau^{-}\right\rangle$, the ratio of the number of growing and shrinking rods $n_{g} / n_{s}$ and $\alpha$ itself. This Figure illustrates Eq. 24

\section{Connections with the individual history of the rods}

On the graph showing the individual history of the rods (Figure 51), one can decompose the time of growth $T^{+}$into a sum of elementary growth intervals $\tau_{j}^{+}$, and the time of shrinking $T^{-}$into a sum of elementary shrinking intervals $\tau_{j}^{-}$:

$$
T^{+}=\sum_{j} \tau_{j}^{+} ; T^{-}=\sum_{j} \tau_{j}^{-} .
$$

In particular, we expect that the following relations between the average elementary time of growth $\left\langle\tau^{+}\right\rangle$, shrinkage $\left\langle\tau^{-}\right\rangle$, and the rates $p_{g s}$ and $p_{s g}$ hold for an isotropic system:

$$
p_{g s}=\left\langle\tau^{+}\right\rangle^{-1} ; p_{s g}=\left\langle\tau^{-}\right\rangle^{-1} .
$$

For an anisotropic system, $p_{g s}$ becomes orientation dependent, while $p_{s g}$ should not. In a stationary case, the following "detailed balance" relation holds:

$$
n_{g} p_{g s}=n_{s} p_{s g}
$$

while in a quasi-stationary state, we expect only a qualitative relation:

$$
\frac{n_{g}}{n_{s}} \simeq \frac{p_{s g}}{p_{g s}}
$$

suggesting the relation:

$$
\frac{n_{g}}{n_{s}} \simeq \frac{p_{s g}}{p_{g s}}=\frac{\left\langle\tau^{+}\right\rangle}{\left\langle\tau^{-}\right\rangle} .
$$

In any case, from Eq. 7 we have:

$$
\begin{aligned}
\langle l\rangle & =\left(v^{+}-v^{-}\right)\left\langle T^{-}\right\rangle+v^{-}\left\langle T^{-}\right\rangle, \\
\langle T\rangle & =\left\langle T^{+}\right\rangle+\left\langle T^{-}\right\rangle .
\end{aligned}
$$

The ratio of growing to shrinking time is

$$
\frac{\left\langle T^{+}\right\rangle}{\left\langle T^{-}\right\rangle}=\alpha\left(\frac{1+\frac{\langle l\rangle}{\alpha v\langle T\rangle}}{1-\frac{\langle l\rangle}{v\langle T\rangle}}\right),
$$

where the r.h.s. is expected to stay close to the value $\alpha$, as $l$ is smaller than $v\left\langle T^{-}\right\rangle$, except for young rods. Because the number of growing time intervals is close to the number of shrinking time intervals, there should not be a large difference between the ratios $\left\langle T^{+}\right\rangle /\left\langle T^{-}\right\rangle$ and $\left\langle\tau^{+}\right\rangle /\left\langle\tau^{-}\right\rangle$, suggesting, along with Eq. 21] another relation:

$$
\frac{\left\langle\tau^{+}\right\rangle}{\left\langle\tau^{-}\right\rangle} \simeq \frac{\left\langle T^{+}\right\rangle}{\left\langle T^{-}\right\rangle}=\frac{n_{g}}{n_{s}} \simeq \alpha .
$$

To test this relation, we performed a set of simulations with the same rate of injection $Q_{i}=1000$, and varying $\alpha$. Both $n_{g}, n_{s},\left\langle\tau^{+}\right\rangle,\left\langle\tau^{-}\right\rangle,\left\langle T^{+}\right\rangle$and $\left\langle T^{-}\right\rangle$can be independently obtained from the simulation, as summarized in Figure 14] The ratio $n_{g} / n_{s}, T^{+} / T^{-}$, and $\left\langle\tau^{+}\right\rangle /\left\langle\tau^{-}\right\rangle$seems to remain remarkably constant as the simulation goes on. The agreement is not quantitative, but the four quantities of relation 24] show significant correlations (Figure 14).

\section{Anisotropic and non-stationary solutions of the kinetic theory}

There are indications that the stationary, homogeneous situation is not the only possible solution of the kinetic theory. We outline in Appendix D the main features of a stationary but anisotropic solution, with a non trivial dependence of $p_{g s}$ in the orientation $\Omega$. This solution can explain why the system tends to choose a preferential global orientation. However, the predicted anisotropy is less than the one that is numerically observed in our system.

The kinetic model described above can also describe time dependent solutions. However, we did not find any simple time-dependent solution compatible with our boundary conditions, i.e. a constant injection rate. It remains that the existence of an unstable, non stationary and anisotropic solution of the kinetic model cannot be ruled out.

\section{CONCLUSION}

We proposed and tested numerically a new paradigm for a kinetically constrained growth mechanism of living rods. We demonstrated that the collective behavior of 
the rods were leading to the formation of bundles of well oriented rods, in the absence of excluded volume interactions and chemical gradients. We suggest this mechanism as a possible alternative in the formation and the orientation of microtubules gels.

We tested the responsive properties of the system to some external stress. We found that the alignment was much faster in the presence of any small anisotropic bias. This result is interesting in the context of the experimental microgravity results of Tabony 30, 31]. We developed a kinetic theory which accounts for the basic scaling properties of the system: conversion rates, average length of the microtubules. The quantitative agreement remains poor, due to the strong correlations present in this two-dimensional system, in which the emergence of large anisotropic domains is incompatible with the homogeneity assumption ( $\vec{r}$ independence).

Because we were able to show that this mechanism also induces some alignment in three dimension, we believe that our kinetic theory would give a better agreement in higher dimensional systems, which is the subject of future work.

\section{APPENDIX A: A KINETIC THEORY FOR HOMOGENEOUS DISTRIBUTIONS}

We assume first that the system is homogeneous, and that the distribution functions do not depend on $\vec{r}$. Denoting by $S$ the area of the system, the distribution functions then reduce to their homogeneous form:

$$
c_{g, s, t}(l, \Omega, \vec{r}, t)=\frac{1}{S} c_{g, s, t}(l, \Omega, t) .
$$

The functions $c_{g, s, t}$ are "extensive" functions of the area $S$. We propose a master equation for these functions, which accounts for the shrinking and growing behavior of the rods, and also account for the possibility of interconversion between the $\mathrm{s}$ and $\mathrm{g}$ states. In order to cope with a possible global anisotropy of the rods, we let the interconversion rates depend on the orientation $\Omega$, but not on the length: $p_{g s}(\Omega) \mathrm{d} t$ is the fraction of $g$-rods which switches to a $s$-rod state during the time interval $\mathrm{d} t$, while $p_{s g}(\Omega) \mathrm{d} t$ describes the reverse change. The corresponding master equation reads:

$$
\left\{\begin{array}{l}
c_{g}(l, \Omega, t+d t)=c_{g}(l-v d t, \Omega, t)+ \\
p_{s g}(\Omega) c_{s}(l, \Omega, t) d t-p_{g s}(\Omega) c_{g}(l, \Omega, t) d t \\
c_{s}(l, \Omega, t+d t)=c_{s}\left(l+v^{-} d t, \Omega, t\right)+ \\
p_{g s}(\Omega) c_{g}(l, \Omega, t) d t-p_{s g}(\Omega) c_{s}(l, \Omega, t) d t
\end{array}\right.
$$

The continuous limit $\mathrm{d} t \rightarrow 0$ leads to a system of two partial differential equations:

$$
\left\{\begin{array}{c}
\frac{\partial c_{g}}{\partial t}+v \frac{\partial c_{g}}{\partial l}=p_{s g} c_{s}-p_{g s} c_{g} \\
\frac{\partial c_{s}}{\partial t}-\alpha v \frac{\partial c_{s}}{\partial l}=p_{g s} c_{g}-p_{s g} c_{s}
\end{array}\right.
$$

where appears the ratio $\alpha=v^{-} / v=v^{-} /\left(v^{+}-v^{-}\right)$.
The partial derivatives in the left hand sides of Eq. A2 correspond to a drift motion of the rods along the $l$ axis. We can associate to this drift the "currents" of growing $j_{g}(l, \Omega, t)=v c_{g}$ and shrinking $j_{s}(l, \Omega, t)=-\alpha v c_{s}$ rods. The sum $j_{g}+j_{s}$ measures the difference between the number of rods which have grown bigger than $l$, and the number of rods which have shrunk below $l$. The distribution $c_{t}=c_{g}+c_{s}$ obeys a usual conservation equation $\partial c_{t} / \partial t+\partial\left(j_{g}+j_{s}\right) / \partial l=0$, provided $l>0$.

In particular, as there is no other option for a rod with length 0 than growing or disappearing, and our master equations must be completed with a boundary condition involving $j_{g}$ and the injection rate $Q_{i}$.

$$
Q_{i}(\Omega)=v c_{g}(l=0, \Omega)
$$

Meanwhile, the rate of disappearance of the rods is $Q_{d}$, obeying

$$
Q_{d}(\Omega)=\alpha v c_{s}(l=0, \Omega)
$$

At the other extreme, we expect that

$$
\lim _{l \rightarrow \infty} c_{g, s, t}(l, \Omega)=0
$$

Equations A2 A3 A5 are the basis of our kinetic theory.

\section{APPENDIX B: THE STATIONARY CASE}

The above system of equations is simpler if we look for a time independent solution, setting the time partial derivative to zero. Introducing $c_{t}=c_{g}+c_{s}$, and $c_{d}=$ $c_{g}-c_{s}$, we get:

$$
\left\{\begin{array}{l}
\frac{v}{2}(1-\alpha) \frac{\partial c_{t}}{\partial l}+\frac{v}{2}(1+\alpha) \frac{\partial c_{d}}{\partial l}=0 \\
\frac{v}{2}(1-\alpha) \frac{\partial c_{d}}{\partial l}+\frac{v}{2}(1+\alpha) \frac{\partial c_{t}}{\partial l}= \\
\left(p_{s g}-p_{g s}\right) c_{t}-\left(p_{s g}+p_{g s}\right) c_{d} \\
c(0, \Omega)=q ; \lim _{l \rightarrow \infty} c(l, \Omega)=0
\end{array}\right.
$$

with solution

$$
\left\{\begin{array}{l}
c_{t}(l, \Omega)=q e^{-l / \bar{l}(\Omega)} \\
c_{d}(l, \Omega)=\frac{\alpha-1}{1+\alpha} q e^{-l / \bar{l}(\Omega)}
\end{array}\right.
$$

where, for convenience, we have introduced $q=c_{t}(0, \Omega)$, while the average length in the direction $\Omega$ is given by:

$$
\bar{l}(\Omega)=\frac{\alpha v}{\alpha p_{g s}(\Omega)-p_{s g}(\Omega)} .
$$

Moreover, the distributions $c_{g}$ and $c_{s}$ verify

$$
\frac{c_{g}(l, \Omega)}{c_{s}(l, \Omega)}=\alpha,
$$


and consequently,

$$
\begin{aligned}
& c_{g}=\frac{\alpha q}{\alpha+1} e^{-l / \bar{l}(\Omega)} \\
& c_{s}=\frac{q}{\alpha+1} e^{-l / \bar{l}(\Omega)} .
\end{aligned}
$$

Finally, the injection rate $Q_{i}(\Omega)$ is related to $q(\Omega)$ by

$$
Q_{i}(\Omega)=\frac{\alpha}{\alpha+1} v q(\Omega)
$$

which is the basis of a stationary, homogeneous solution of the system, expressed in terms of $Q_{i}, \alpha, p_{g s}$ and $p_{s g}$. An example of explicit angular dependence of $Q_{i}(\Omega)$ is the situation described in section IIIB and in Figure 4 In most cases, however, we are interested in an isotropic, constant, function $Q_{i}$, which we consider now.

To move further, we must estimate the interconversion rates $p_{g s}$ and $p_{s g}$. To estimate $p_{s g}$, we assume that collisions are pairwise, and, as in the usual kinetic theory of gases, that there is no correlation between any two colliding rods. Then, $p_{s g}$ does not depend on angles, and is inversely proportional to the average waiting time $\left\langle\tau^{-}\right\rangle$ spent in the blocked state. Since all rods shrink at the same speed, the waiting time depends only on the distance between the contact point and the minus end of the restricting rod. Assuming a uniform distribution of contact points along the rod, we find that the average waiting time associated to a restricting rod with length $l$ is $l /\left(2 v^{-}\right)$, and consequently,

$$
\left\langle\tau^{-}\right\rangle=\frac{\bar{l}}{2 v^{-}} ; p_{s g}=\frac{1}{\left\langle\tau^{-}\right\rangle}=\frac{2 v^{-}}{\bar{l}} .
$$

In this equation, we need to know the average length $\bar{l}$ of the rods, irrespective of their orientation. Given the solution obtained above, this simply reads:

$$
\bar{l}=\frac{\int \mathrm{d} l \mathrm{~d} \Omega l c_{t}(l, \Omega)}{\int \mathrm{d} l \mathrm{~d} \Omega c_{t}(l, \Omega)}=\frac{\int \mathrm{d} \Omega(\bar{l}(\Omega))^{2}}{\int \mathrm{d} \Omega \bar{l}(\Omega)} .
$$

The estimate of $p_{g s}$ also comes from the analogy with the kinetic theory of gases. We imagine the system from the point of view of an observer sitting at the top of a growing tip, and estimate the area swept by the mesh of all the other rods, moving relatively to the observer at speed $-v^{+}$. The typical collision time is reached when this area becomes comparable to the total area $S$ of the system, making the probability of collision of order one. The calculation shows that the collision time depends on the projected length $l^{\prime}$ of the obstructing rod, and on the relative orientation difference $\Omega-\Omega^{\prime}$ between the two rods. We can write:

$$
\overline{l_{p}}(\Omega)=\frac{\int d \Omega^{\prime} d l^{\prime} l^{\prime}\left|\sin \left(\Omega-\Omega^{\prime}\right)\right| c_{t}\left(l^{\prime}, \Omega^{\prime}\right)}{\int d \Omega^{\prime} d l^{\prime} c_{t}\left(l^{\prime}, \Omega^{\prime}\right)},
$$

and the probability $p_{g s}(\Omega)$ reduces to:

$$
p_{g s}(\Omega)=v^{+} \overline{l_{p}}(\Omega)\left(\frac{n_{t}}{S}\right)
$$

where $S$ is the total area of the system, $n_{t}$ is the total number of rods, and $\left(n_{t} / S\right)$ is the ratio of two "extensive" functions.

\section{APPENDIX C: THE ISOTROPIC SOLUTION AND ITS PREDICTIONS}

The isotropic hypothesis consists in taking $p_{g s}$ and $p_{s g}$ angle independent. This simplifies the above kinetic theory to a point where a self-consistent analytical solution becomes available. The $\Omega$ dependence of $\bar{l}$ and $\bar{l}_{p}$ drops out, and we get:

$$
\bar{l}_{p}=\frac{2 \bar{l}}{\pi}
$$

and equation $\$ 2$ leads to the self-consistence relations:

$$
\begin{aligned}
\bar{l} & =\frac{\alpha v}{\alpha v^{+} \frac{n_{t}}{S} \frac{2 \bar{l}}{\pi}-\frac{2 v^{-}}{\bar{l}}} \\
(\bar{l})^{2} & =\frac{3 \pi}{2(\alpha+1)} \frac{S}{n_{t}}
\end{aligned}
$$

Then, we replace $n_{t}$ by $\frac{\alpha+1}{\alpha} \frac{Q_{i} \bar{l}}{v}$, to make a prediction for the average length $\bar{l}$ and the number of rods $n_{t}$.

$$
\begin{aligned}
\bar{l} & =\sqrt{\frac{3 \pi}{2(\alpha+1)} \frac{S}{n_{t}}} \simeq\left(\frac{S}{n_{t}}\right)^{\frac{1}{2}} \\
\bar{l} & =\sqrt[3]{\frac{3 \alpha}{4(\alpha+1)^{2}} \frac{S v}{Q_{i}}} \simeq\left(\frac{S v}{Q_{i}}\right)^{\frac{1}{3}} \\
n_{t} & =2 \pi \frac{\alpha+1}{\alpha v} \bar{l} Q_{i} .
\end{aligned}
$$

\section{APPENDIX D: THE ANISOTROPIC SOLUTION}

We believe that the system of equations B2, B6. B7. B8. B9 also admits an anisotropic solution, characterized by an explicit $\Omega$ dependence of $p_{g s}(\Omega)$ and $\bar{l}(\Omega)$ while $p_{s g}$ remains isotropic. To approach this solution, we expand $\bar{l}(\Omega)$ in cosine series:

$$
\bar{l}(\Omega)=l_{0}+l_{2} \cos (2 \Omega)+l_{4} \cos (4 \Omega) \ldots
$$

and approximate $\sin \left|\Omega-\Omega^{\prime}\right|$ in a similar manner:

$$
\sin |\Omega|=s_{0}+s_{2} \cos (2 \Omega)+s_{4} \cos (4 \Omega) \ldots
$$

Possible choices include the Fourier expansion:

$$
\sin |\Omega|=\frac{2}{\pi}-\frac{4}{\pi} \sum_{p=1}^{\infty} \frac{\cos (2 p \Omega)}{4 p^{2}-1}
$$

or replacing $\sin |\Omega|$ by $\sin ^{2}(\Omega)$. 
For instance, by keeping the two first terms in the expansion, and writing the self-consistence equation B2 under the form $\bar{l}(\Omega) \times\left(\alpha p_{g s}(\Omega)-p_{s g}\right)=\alpha v$, we finally obtain a system of equations for $l_{0}$ and $x=l_{2} / l_{0}$ :

$$
\begin{aligned}
& \frac{\alpha v^{+} n_{t} l_{0}^{2}}{S}\left(s_{0}\left[1+\frac{x^{2}}{2}\right]+s_{2} \frac{x^{2}}{2}\right)=3 v^{-} \\
& \frac{\alpha v^{+} n_{t} l_{0}^{2}}{S}\left(s_{0} x\left[1+\frac{x^{2}}{2}\right]+s_{2} x\right)=\frac{2 v^{-} x}{1+\frac{x^{2}}{2}} .
\end{aligned}
$$

We observe that the isotropic solution $x=0, l_{0}^{2}=$ $3 S v^{-} /\left(s_{0} \alpha v^{+} n_{t}\right)$ (equivalent to C3) coexists along with an anisotropic solution $x \neq 0$, where $x$ solves

$$
\frac{\alpha v^{+} n_{t} l_{0}^{2}}{S}\left(s_{0}\left[1+\frac{x^{2}}{2}\right]+s_{2}\right)=\frac{2 v^{-}}{1+\frac{x^{2}}{2}},
$$

and $l_{0}$ is a function of $x$ and the other parameters of the problem.

Thus, despite its strong mean field features, the kinetic model is compatible with the emergence of an anisotropic solution, with an explicit angular dependence of the average length of the rods. However, this anisotropy is bounded, with an average length finite in all directions, while the domains observed in the simulations can grow without limit.

\section{ACKNOWLEDGMENTS}

The authors wish to thank Professor J. Tabony for discussions inspired this work. V. B. gratefully acknowledges Centre National d'Etudes Spatiales (CNES) for a research post-doctoral fellowship.
[1] Howard, J., 2001, Mechanics of motor proteins and the cytoskeleton, Sinauer Associates Inc. Sunderland.

[2] Gadde, S., and R. Heald, 2004, Mechanisms and molecules of the review mitotic spindle, Current Biology, 14: R797-R805.

[3] Hyman, A. A., and E. Karsenti, 1996, Morphogenetic properties of microtubules and mitotic spindle assembly, Cell, 84: 401-410.

[4] Desai, A., and T. J. Mitchison, 1997, Microtubule polymerization dynamics, Annu. Rev. Cell Dev. Biol. 13: 83117.

[5] Valiron, O., N. Caudron, and D. Job, 2001, Microtubule dynamics, CMLS, 58: 2069-2084.

[6] Canaday, J., V. Stoppin-Mellet, J. Mutterer, A. M. Lambert, and A. C. Schmit, 2000, Higher plant cells: Gamma-tubulin and microtubule nucleation in the absence of centrosomes. Microsc. Res. Tech. 49: 487-495.

[7] Murata, T., S. Sonobe, T. I. Baskin, S. Hyodo, S. Hasezawa, T. Nagata, T. Horio, and M. Hasebe, 2005, Microtubule-dependent microtubule nucleation based on recruitment of $\gamma$-tubulin in higher plants, Nature Cell Biol, 7: 961-968.

[8] Vos, J. W., M. Dogterom, and A. M. C. Emons, 2004, Microtubules become more dynamic but not shorter during preprophase band formation: a possible "search-andcapture" mechanism for microtubule translocation, Cell Motility and the Cytosk., 57: 246-258.

[9] Maly, I. E., 2002, Diffusion Approximation of the Stochastic Process of Microtubule Assembly, Bulletin of Mathematical Biology 64: 213-238.

[10] Janosi, I. M., D. Chretien, and H. Flyvbjerg, 2002, Structural microtubule cap: stability, catastrophe, rescue, and third state, Biophys. J., 83: 1317-1330.

[11] Krebs, A., K. N. Goldie, and A. Hoenger, 2005, Structural rearrangements in tubulin following microtubule formation, EMBO reports, 6: 227-232.

[12] Janson, M. E., M. E. de Dood, and M. Dogterom, 2003, Dynamic instability of microtubules is regulated by force, J. Cell Biol., 161: 1029-1034.
[13] Tran, P. T., L. Marsh, V. Doye, S. Inoue, and F. Chang, 2001, A mechanism for nuclear positioning in fission yeast based on microtubule pushing, J. Cell Biol., 153: 397411.

[14] Dogterom, M., and B. Yurke, 1997, Measurement of the force-velocity relation for growing microtubules, Science, 278: 856-860.

[15] Dogterom, M., M. E. Janson, C. Faivre-Moskalenko, A. van der Horst, J. W. J. Kerssemakers, C. Tanase, and B. M. Mulder, 2002, Force generation by polymerizing microtubules, Appl. Phys. A, 75: 331-336.

[16] Dhonukshe, P., and Jr. T. W. J. Gadella, 2003, Alteration of microtubule dynamic instability during preprophase band formation revealed by yellow fluorescent ProteinCLIP170 Microtubule plus-end labeling, The Plant Cell, 15: 597-611.

[17] Bastiaens, P., M. Caudron, P. Niethammer, and E. Karsenti, 2006, Gradients in the self-organization of the mitotic spindle, TRENDS in Cell Biology, 16(3): 125134.

[18] Dixit, R., and R. Cyr, 2004. Encounters between dynamic cortical microtubules promote ordering of the cortical array through angle-dependent modifications of microtubule behavior, The Plant Cell. 16: 3274-3284.

[19] Tabony, J., and D. Job, 1992, Gravitational symmetry breaking in microtubular dissipative structures, Proc. Natl. Acad. Sci. USA, 89: 6948-6952.

[20] Tabony, J., 1996, Self-organization in a simple biological system through chemically dissipative processes, Nanobiology, 04: 117-137.

[21] Glade, N., and J. Tabony, 2005, Brief exposure to high magnetic fields determines microtubule self-organisation by reaction-diffusion processes, Biophysical Chemistry 115: 29-35.

[22] van Bruaene, N., G. Joss, and P. van Oostveldt, 2004, Plant Physiology, 136: 3905-3919.

[23] Wymer, C. L., D. D. Fisher, R. C. Moore, and R. J. Cyr, 1996, Elucidating the mechanism of cortical microtubule reorientation in plant cells, Cell Motility and the Cytosk., 
35: $162-173$.

[24] Baulin, V. A., and A. R. Khokhlov, 1999, Nematic ordering of rigid rods in a gravitational field, Phys. Rev. E, 60: 2973-2977.

[25] Baulin, V. A., 2003, Self-assembled aggregates in the gravitational field: Growth and nematic order, J. Chem. Phys., 119: 2874-2885.

[26] Himmelspach, R., C. Wymer, C. W. Lloyd, and P. Nick, 1999, Gravity-induced reorientation of cortical microtubules observed in vivo, The Plant J., 18: 449-453.

[27] Lloyd, C. W., R. Himmelspach, P. Nick, and C. Wymer, 2000, Cortical microtubules form a dynamic mechanism that helps regulate the direction of plant growth, Grav.
Space Biol. Bull., 13: 59-66.

[28] Wasteneys ,G. O., 2002, Microtubule organization in the green kingdom: chaos or self-order?, J. Cell Sci., 115: 1345-1354.

[29] Bray, A.J., 1994, Theory of phase ordering kinetics, Advances in Physics, vol: 358.

[30] Tabony, J., N. Glade, J. Demongeot, and C. Papaseit, 2002, Biological Self-Organization by Way of Microtubule Reaction-Diffusion Processes, Langmuir, 18: 7196-7207.

[31] Glade, N., J. Demongeot, and J. Tabony, 2002, Comparison of reaction-diffusion simulations with experiment in self-organized microtubule solutions, C. R. Biologies, 325: 283-294. 